\title{
Correction to: Cholesterol Effects on the Physical Properties of Lipid Membranes Viewed by Solid-state NMR Spectroscopy
}

Trivikram R. Molugu and Michael F. Brown

\section{Correction to:}

Chapter 5 in: A. Rosenhouse-Dantsker, A. N. Bukiya (eds.), Cholesterol Modulation of Protein Function, Advances in Experimental Medicine and Biology 1115, https://doi.org/10.1007/978-3-030-04278-3_5

Chapter 5 was published without including the research grant details in the acknowledgement section, which has been updated in the chapter now. 